\title{
Evaluación del control de la presión arterial y la adherencia terapéutica en hipertensos seguidos en el Programa de Salud Cardiovascular (PSCV). Asociación con características clínicas, socioeconómicas y psicosociales.
}

\author{
Javier Chacón ${ }^{1}$, Daniela Sandoval ${ }^{1}$, Reinaldo Muñoz ${ }^{1}$, Tomás Romero². \\ 1 Departamento de Atención Primaria y Salud Familiar, Facultad de \\ Medicina, Universidad de Chile, Santiago, Chile. \\ 2 School of Medicine, University of California, San Diego, California, USA, \\ Institutional Review Board, Sharp Health Care, San Diego, California.
}

Antecedentes: Inadecuado control de presión arterial (PA) y baja adherencia a tratamiento farmacológico $(\mathrm{Rx})$ en hipertensos son problemas persistentes globales y en Chile. Factores socioeconómicos y psicosociales han sido frecuentemente mencionados, pero escasamente en Chile.

Objetivo: Evaluar control de la PA y adherencia a Rx en hipertensos seguidos en el Programa de Salud Cardiovascular (PSCV) y su asociación con factores clínicos, socioeconómicos y psicosociales.

Métodos: Muestra randomizada de 1.794 hipertensos seguidos por 1 año en PSCV en Región Metropolitana. Se evaluó la asociación de edad, sexo, educación, ingreso familiar, Rx, diabetes, obesidad, tabaquismo, consumo problemático de alcohol y actividad física con el control de la PA $(<140 / 90$ $\mathrm{mmHg}$ ) y adherencia. Además, en 600 pacientes, se evaluó la asociación con estrés emocional/depresión, relación médico-paciente y apoyo social. Se obtuvieron Odds Ratio (OR) mediante análisis de regresión logística multivariante.

Resultados: PAcontrolada se comprobó en 56,5\% y adherencia en $37,3 \%$ sin documentarse asociación entre ambas (OR 1,01 [IC 95\% 0,78-1,32]). Factores asociados a PA no controlada y no adherencia fueron: edad, bajo ingreso familiar, inadecuada relación medico-paciente y alto nivel de estrés emocional/depresión. Rx múltiple y obesidad se asociaron a PA no controlada; sexo masculino y baja educación a no adherencia.

Conclusiones: El control de la PA $(56,5 \%)$ fue similar a resultados de países desarrollados y supera ampliamente cifras de la Encuesta Nacional de Salud $2010(16,9 \%)$. Estos resultados y la falta de asociación entre el control de la PA y la adherencia, sugieren la favorable influencia de otros factores posiblemente relacionados al PSCV.

\section{Correspondencia:}

Javier Chacón Rivas.

Departamento de Atención Primaria y Salud Familiar,

Facultad de Medicina, Universidad de Chile. Gran Avenida

3100, San Miguel, Santiago, Chile.

Código Postal 8900085.

Teléfono: (56-2) 25552716

Fax: (56-2) 5563211.

javierchacon@med.uchile.cl 


\section{Assessment of blood pressure control and adherence in hypertensive patients followed in the Cardiovascular Health Program (CHP). Association with clinical, socioeconomic and psychosocial characteristics}

Background: Unsatisfactory blood pressure (BP) control and low adherence to antihypertensive pharmacotherapy $(\mathrm{Rx})$ in hypertensive populations are persistent problems worldwide and also in Chile. Socioeconomic and psychosocial factors have been frequently mentioned, but with limited contributions from Chile.

Objective: The assessment of BP control and adherence to $\mathrm{Rx}$ in hypertensive patients followed in the Cardiovascular Health Program (CVHP) and to determine their association with clinical, socioeconomic and psychosocial characteristics.

Methods: A randomized sample of 1,794 hypertensive patients followed for 1 year under the CVHP in Metropolitan Region. Association of BP control $(<140 / 90 \mathrm{mmHg})$ and adherence with age, gender, education, income, Rx, diabetes, obesity, smoking, alcohol use problem and physical activity were analyzed. In a subgroup of 600 patients additional analysis included the association with emotional stress and depression, patient-physician relation and social support. Odds Ratio (OR) were obtained by multivariate logistic regression.

Results: BP control was achieved in $56.5 \%$ and adherence in $37.3 \%$. No association was found between them (OR 1.01 [CI 95\% 0.78-1.32]). Uncontrolled BP and no adherence were associated to advanced age, low income, poor patient-physician relation and high stress-depression. Obesity and multiple Rx were associated to uncontrolled BP. Male gender and low education, were associated to no adherence.

Conclusions: BP control (56.5\%) was similar to results obtained in developed countries and is strikingly higher than the results reported in the 2010 Chilean National Health Survey (16.9\%). These results and the lack of association between BP control and adherence suggest the favorable influence of unaccounted factors, possibly related to the CVHP.

Key words: Hypertension, blood pressure control, adherence to medication. 


\section{Introducción:}

El control insatisfactorio de la presión arterial (PA) de acuerdo a lo recomendado por las guías clínicas de uso actual $(<140 / 90 \mathrm{mmHg})$ es un problema persistente en la población hipertensa. En los países desarrollados y de alto ingreso cerca del $75 \%$ de los hipertensos tienen acceso a la atención médica en contraste con menos del $30 \%$ en la mayoría de los países en desarrollo y bajo ingreso. Aproximadamente 55\% de los pacientes con acceso a tratamiento farmacológico antihipertensivo (Rx) no logran un control satisfactorio de la PA. La baja adherencia a Rx es considerado como un factor importante de esta discordancia ${ }^{1-3}$. Factores socioeconómicos y psicosociales han sido frecuentemente mencionados como una barrera en el logro del control de la PA y la adherencia a Rx, de particular relevancia en países en desarrollo y/o en estratos sociales con limitado acceso a la atención de salud ${ }^{4-6}$. En el año 2002, el Ministerio de Salud puso en marcha el Programa de Salud Cardiovascular (PSCV). Uno de los objetivos de este programa de libre acceso, que funciona en los centros de Atención Primaria de Salud (APS), es el tratamiento y la detección de factores de riesgo cardiovascular asociados en los pacientes hipertensos, mediante un manejo integral con enfoque multidisciplinario destinado a modificar estilos de vida y hábitos desfavorables con seguimiento periódico y que incluye el Rx. El PSCV fue iniciado con el objetivo de reducir la morbi-mortalidad cardiovascular, actualmente la mayor causa de muerte y segunda causa de discapacidad en Chile 7,8 .

En 2009 comenzamos el seguimiento de una cohorte de estos pacientes con el objetivo de analizar factores clínicos y socioeconómicos relacionados con el control de la PA. Nuestra hipótesis fue que la eliminación de algunos obstáculos en el acceso a la atención de salud logrados por el PSCV permitiría lograr una mejoría en el control de la PA en los pacientes hipertensos. Resultados iniciales de este estudio ya han sido comunicados y publicados anteriormente ${ }^{6,9}$.

El objetivo del presente estudio es proporcionar evidencias adicionales a nuestros hallazgos preliminares utilizando una base de datos ampliada que incluye un subgrupo de pacientes $(n=600)$ en los que se evaluó la asociación de factores psicosociales y hábitos de estilo de vida con el control de la PA y la adherencia a Rx. Hasta donde nos ha sido posible comprobar no existen estudios similares publicados en Chile o en países de la Región Latinoamericana.

\section{Métodos}

\section{Población}

El estudio fue conducido en centros de APS con PSCV de la Región Metropolitana de Chile. Los pacientes en los centros de APS con PA $\geq 140 / 90 \mathrm{mmHg}$ son derivados al PSCV donde se les realiza una evaluación de factores de riesgo asociados (diabetes, dislipidemias, insuficiencia renal) y son seguidos cada 3 meses aproximadamente por un equipo de salud que incluye: médicos de APS, nutricionistas y personal de salud entrenados que proveen evaluación clínica y de hábitos y estilos de vida otorgando educación y recomendaciones para la modificación de conductas poco saludables.

\section{Muestra}

De un total de 153 centros de APS con PSCV de la Región Metropolitana, se seleccionó en forma aleatoria 52 centros, considerando un centro de APS por comuna con más de 1.000 hipertensos bajo control. Se estimó una muestra aleatoria simple de 1.194 pacientes que provinieron proporcionalmente de los 52 centros de APS seleccionados. El tamaño de la muestra se obtuvo con un nivel de confianza de $95 \%$, error estimado de $5 \%$, prevalencia esperada de control de la PA $(<140 / 90 \mathrm{mmHg})$ de $50 \%$ y efecto de diseño de 3 . En estos pacientes se evaluaron características socioeconómicas, clínicas y antropométricas que se describen más adelante.

La evaluación de la adherencia a tratamiento antihipertensivo y las características psicosociales y de estilos de vida desfavorables fue realizado en un nuevo grupo de 600 pacientes hipertensos referidos al PSCV en 4 de los mismos centros de APS seleccionados en el grupo inicial (1.194 pacientes). Este nuevo grupo fue añadido al grupo anterior alcanzando un total de 1.794 sujetos con el propósito de analizar las características demográficas y clínicas.

En ambos grupos fueron incluidos pacientes hipertensos que participaban en el PSCV por más de 1 año. Hipertensos menores de 20 años de edad o con discapacidades significativas (postrados, usuarios de silla de rueda y mentalmente deshabilitados) o con inasistencias frecuentes e injustificadas a sus controles de rutina fueron excluidos.

\section{Procedimientos}

Se revisaron los registros médicos de 12 meses consecutivos, donde se recogió la información clínica y demográfica pertinente. Los valores de PA fueron registrados en cada una de las visitas del paciente después de tres mediciones sucesivas con el paciente sentado, al menos durante $5 \mathrm{mi}-$ nutos, usando esfigmomanómetro de mercurio. Un valor de $\mathrm{PA}<140 / 90 \mathrm{mmHg}$ fue considerado como indicativo de un control satisfactorio.

La presencia de diabetes y otras comorbilidades fueron es- 
tablecidas mediante el diagnóstico efectuado por un médico y/o por la terapia farmacológica prescrita. Los pacientes con comorbilidades cardiovasculares asociadas o aquellos que requieren hemodiálisis son habitualmente remitidos al nivel secundario de atención y no fueron incluidos en este estudio. Exámenes de laboratorio (glucosa, hemoglobina glicosilada [HbA1c], colesterol total y HDL) fueron medidos usando técnicas estándar. Se registraron todos los medicamentos antihipertensivos en uso al final del período de 12 meses de seguimiento en el PSCV. Los pacientes fueron citados a una sesión especial para la medición de variables antropométricas (peso, talla, circunferencia de cintura), utilizando procedimientos estandarizados previamente descritos ${ }^{6,9}$. Personal capacitado informó a los pacientes sobre cuestionarios previamente validados en población chilena sobre hábitos de estilo de vida, características socioeconómicas y psicosociales. Los pacientes llenaron estos cuestionarios después de haber completado al menos 6 meses en el PSCV en casa o durante sus citas mientras esperaban en la sala de espera. A todos los pacientes se les dio asistencia para completar los cuestionarios cuando así lo requirieron.

Bajo ingreso familiar se definió como menor que el cuartil más bajo de ingresos familiares mensuales por persona ( $<45.000$ pesos por persona). Baja educación correspondió a una educación menor de 8 años de estudios aprobados. La evaluación de la adherencia a tratamiento antihipertensivo, características psicosociales y de hábitos de estilo de vida desfavorables se evaluó en un nuevo grupo de 600 pacientes.

Medición de las características psicosociales. Para ello se utilizaron diversos tests, a saber:

1. Test Morisky-Green-Levinne (MGL-4), se utilizó para evaluar la adherencia a $\mathrm{Rx}^{10}$. Esta prueba tiene una comprobada validez predictiva en el control de la PA a 5 años ${ }^{11}$. Detalles de este cuestionario han sido comunicados anteriormente ${ }^{9,10,11}$.

2. Cuestionario de Red de Apoyo social (MOS-SS) ${ }^{12}$, adaptado al español y validado para su uso en APS chilena. Recoge información multidimensional acerca de los niveles de apoyo a los que el paciente puede acceder. Escaso apoyo social se definió con una puntuación en escala de Likert < 57 puntos (máximo de 94).

3. Score de estrés emocional y depresión, se utilizó la versión en español y validada en Chile del cuestionario GHQ-12 ${ }^{13}$ que detecta y gradúa los niveles de estrés emo- cional y depresión. La puntuación más alta es de 12 puntos; una puntuación de 7 o más refleja un alto grado de estrés emocional y depresión.

4. Relación médico paciente, se utilizó la versión validada del cuestionario chileno realizado por Bozzo-Martínez ${ }^{14}$ para evaluar la satisfacción del paciente con la atención recibida en los centros de APS. Esta versión cuenta con 21 declaraciones que cubren tres aspectos de la relación del paciente con su proveedor de atención. Detalles de este cuestionario pueden consultarse en publicaciones previas $^{9,14}$. El paciente responde cada aseveración con una puntuación de 1 a 5 puntos (según escala de Likert). En nuestro estudio se validó el cuestionario (alfa de Cronbach 0,95). Una puntuación menor de 71 puntos se consideró como indicativo de una relación médico paciente inadecuada.

5. Hábito tabáquico, los fumadores fueron definidos como aquellos que eran fumadores activos al momento del estudio.

6. Consumo de alcohol, se evaluó a través de la encuesta EBBA $^{15}$ (escala breve del beber anormal) para identificar comportamiento anormal relacionado con el alcohol. Esta encuesta fue previamente validada y es de uso común en Chile $^{15}$. La encuesta consta de 7 preguntas relacionadas con patrones de consumo de alcohol. Dos o más respuestas positivas identifican un uso problemático del alcohol.

7. Actividad física, los pacientes físicamente activos se definieron como aquellos que efectúan ejercicios en forma electiva (no relacionados con sus actividades laborales) durante 30 minutos o más al menos 3 veces por semana, utilizando los criterios empleados por la Encuesta Nacional de Salud ${ }^{16}$.

El protocolo de este estudio fue aprobado por el Comité de Ética en Investigación en Seres Humanos de la Universidad de Chile. Todos los pacientes firmaron consentimiento informado previo al inicio del estudio. No hubo rechazos entre los pacientes seleccionados para participar en el estudio.

\section{Análisis estadístico.}

Se utilizó el programa SPSS (versión 15) para el análisis de datos. Se incluyó un análisis descriptivo de las características demográficas, socioeconómicas, clínicas, antropométricas, adherencia a Rx y hábitos de estilos de vida. Diferencias respecto a sexo para las variables categóricas 
se analizaron mediante test de $\mathrm{X}^{2} \mathrm{y}$ t-student para las variables continuas. Se realizó análisis de regresión logística multivariante para calcular Odds Ratios (OR) con intervalos de confianza de 95\% (IC 95\%) para el control de la PA y para la adherencia a Rx, considerando Modelo 1 ajustado por edad y sexo, y Modelo 2 ajustado por todas las variables medidas.

\section{Resultados}

1. Características socioeconómicas, clínicas, antropométricas y terapia antihipertensiva (Tabla 1)

En esta cohorte se observó una mayor proporción de mujeres $(n=1.262)$ que de hombres $(n=532)$. Bajo ingreso fa- miliar y baja educación fue documentada en aproximadamente $1 / 3$ de los pacientes. Obesidad (IMC $>30 \mathrm{Kg} / \mathrm{m} 2$ ) se presentó en $46,8 \%$ de los pacientes (50,3\% en mujeres), y $28,9 \%$ eran diabéticos (sin diferencias por sexo). El promedio de PA inicial fue $148,9 / 95,8 \mathrm{mmHg}$ y $135,7 / 80,9$ mmHg después de un año de seguimiento en el PSCV $(\mathrm{p}<0,001)$. Control satisfactorio de $\mathrm{PA}(<140 / 90 \mathrm{mmHg})$ se observó en $56,5 \%$ de los pacientes, mayor en mujeres $(58,9$ vs $50,8 \%, \mathrm{p}<0,001$ ). La adherencia a Rx (evaluado en el grupo de 600 pacientes) fue $37,3 \%$, mayor en mujeres que en hombres ( $39,9 \%$ vs. $29,8 \%, \mathrm{p}<0,02)$. Consumo problemático del alcohol fue más frecuente en hombres. No se observó diferencias en el consumo de tabaco y estilo de

Tabla 1. Características demográficas, socioeconómicas, clínicas, antropométricas, tratamiento antihipertensivo, hábitos y de estilos de vida en 1.794 pacientes hipertensos seguidos en el Programa de Salud Cardiovascular en la Región Metropolitana, Santiago, Chile.

\begin{tabular}{|c|c|c|c|c|}
\hline & $\begin{array}{r}\text { Total } \\
(n=1.794)\end{array}$ & $\begin{array}{l}\text { Hombres } \\
(n=532)\end{array}$ & $\begin{array}{l}\text { Mujeres } \\
(n=1.262)\end{array}$ & \\
\hline Características & $\mathrm{n}(\%)$ & $\mathrm{n}(\%)$ & $\mathrm{n}(\%)$ & $\mathrm{p}$-value \\
\hline \multicolumn{5}{|l|}{ Demográficas y socioeconómicas } \\
\hline Edad (años)a & $60,5 \pm 12,6$ & $61,7 \pm 12,2$ & $60,0 \pm 12,7$ & 0,01 \\
\hline Baja educación (<8 años de educación) & $644(35,9)$ & $186(34,9)$ & $457(36,2)$ & 0,61 \\
\hline Bajo ingreso familiar ( $<\$ 45,000$ por persona/mes) $\ddagger$ & $214(35,7)$ & $38(24,8)$ & $176(39,4)$ & $<0,001$ \\
\hline \multicolumn{5}{|l|}{ Clínicas } \\
\hline Presión arterial sistólica $(\mathrm{mmHg})^{\mathrm{a}}$ & $135,7 \pm 16,3$ & $137,8 \pm 16,3$ & $134,8 \pm 16,1$ & $<0,001$ \\
\hline Presión arterial diastólica $(\mathrm{mmHg})^{\mathrm{a}}$ & $80,9 \pm 9,1$ & $82,1 \pm 9,7$ & $80,5 \pm 8,9$ & 0,001 \\
\hline Presión arterial controlada (PA<140/90 mmHg) & $1,013(56,5)$ & $270(50,8)$ & $743(58,9)$ & $<0,001$ \\
\hline Glicemia (mg/dL) $)^{a}$ & $112,6 \pm 46,0$ & $114,7 \pm 49,1$ & $111,6 \pm 44,6$ & 0,25 \\
\hline Diabetes Mellitus (diagnostico clínico) & $519(28,9)$ & $162(30,5)$ & $357(28,3)$ & 0,35 \\
\hline Colesterol total $(\mathrm{mg} / \mathrm{dL})^{\mathrm{a}}$ & $210,4 \pm 45,1$ & $200,4 \pm 4$ & $214,8 \pm 46,4$ & $<0,001$ \\
\hline Colesterol total $\geq 240 \mathrm{mg} / \mathrm{dL}$ & $406(22,6)$ & $94(17,7)$ & $312(24,7)$ & 0,001 \\
\hline Colesterol HDL (mg/dL) ${ }^{\mathrm{a}}$ & $48,6 \pm 12,4$ & $44,5 \pm 10,9$ & $50,4 \pm 12,6$ & $<0,001$ \\
\hline Colesterol HDL $<40 \mathrm{mg} / \mathrm{dL}$ & -- & $179(33,6)$ & -- & \\
\hline Colesterol $\mathrm{HDL}<50 \mathrm{mg} / \mathrm{dL}$ & -- & -- & $658(52,1)$ & \\
\hline \multicolumn{5}{|l|}{ Tratamiento Antihipertensivo } \\
\hline No farmacológico & $142(7,9)$ & $26(4,9)$ & $116(9,2)$ & 0,002 \\
\hline Farmacológico & $1,652(92,1)$ & $506(95,1)$ & $1,146(90,8)$ & 0,002 \\
\hline Monoterapia & $697(42,2)$ & $241(47,6)$ & $456(39,8)$ & $<0,001$ \\
\hline Combinación farmacológica & $955(57,8)$ & $265(52,4)$ & $690(60,2)$ & $<0,001$ \\
\hline Adherencia a terapia farmacológica $\ddagger$ & $224(37,3)$ & $46(29,8)$ & $178(39,9)$ & 0,02 \\
\hline \multicolumn{5}{|l|}{ Antropométricas } \\
\hline Peso $(\mathrm{Kg})^{\mathrm{a}}$ & $75,1 \pm 15,2$ & $80,5 \pm 14,4$ & $72,8 \pm 15,0$ & $<0,001$ \\
\hline Altura $\left(m^{a}\right.$ & $1,57 \pm 0,08$ & $1,66 \pm 0,08$ & $1,53 \pm 0,07$ & $<0,001$ \\
\hline Índice masa corporal $(\mathrm{Kg} / \mathrm{m} 2)^{\mathrm{a}}$ & $30,4 \pm 5,5$ & $29,2 \pm 4,4$ & $30,9 \pm 5,9$ & $<0,001$ \\
\hline Obesidad (IMC $\geq 30 \mathrm{Kg} / \mathrm{m} 2)$ & $840(46,8)$ & $199(37,4)$ & $641(50,8)$ & $<0,001$ \\
\hline \multicolumn{5}{|l|}{ Hábitos y estilos de vida } \\
\hline Tabaquismo (fumador actual) $\ddagger$ & $158(26,4)$ & $38(24,8)$ & $120(26,9)$ & 0,58 \\
\hline Consumo problemático de alcoholł & $81(13,5)$ & $48(31,3)$ & $31(7,1)$ & $<0,001$ \\
\hline \multicolumn{5}{|l|}{ Sedentarismo (<30 min $\times 3$ días/semana de actividad } \\
\hline física electiva) $\ddagger$ & $521(86,9)$ & $134(86,9)$ & $387(86,9)$ & 0,99 \\
\hline
\end{tabular}

* Valores expresados en media \pm desviación estándar; $\$ n=600$ pacientes. 


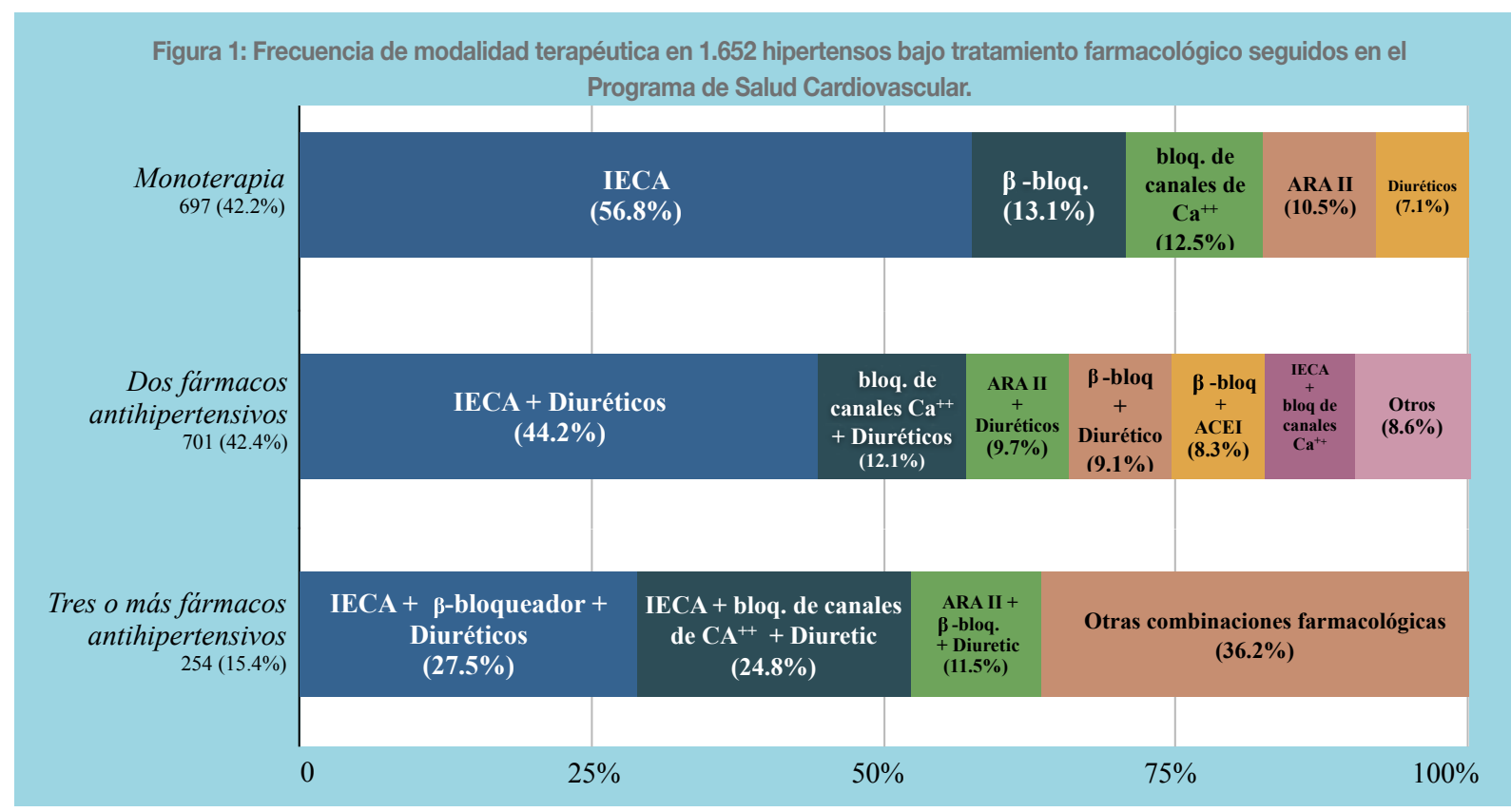

vida sedentario ( $26,4 \%$ y $86,3 \%$ respectivamente). $92,1 \%$ de los pacientes fue tratado con fármacos antihipertensivos, 42,2\% con monoterapia, más frecuentemente inhibidor de la enzima convertidora de angiotensina (IECA) (detalles de fármacos de uso frecuente se muestran en la Figura 1).
2. Factores asociados al control de la PA. (Tabla 2)

Se realizó análisis de regresión logística multivariante en el subgrupo de 600 pacientes para calcular OR y evaluar el riesgo de PA no controlada ( $\geq 140 / 90 \mathrm{mmHg})$. La Tabla 2 muestra OR no ajustados y ajustados usando Modelo 1

Tabla 2: Análisis multivariado para control insatisfactorio de la PA ( $\geq 140 / 90 \mathrm{mmHg})$ en 600 pacientes hipertensos seguidos en el Programa de Salud Cardiovascular en la Región Metropolitana, Santiago, Chile.

\begin{tabular}{|c|c|c|c|c|c|}
\hline Variables & $\begin{array}{c}\mathrm{PA}<140 / 90 \\
\mathrm{mmHg} \\
\mathrm{N}=293 \\
\mathrm{n}(\%)\end{array}$ & $\begin{array}{c}\mathrm{PA} \geq 140 / 90 \\
\mathrm{mmHg} \\
\mathrm{N}=307 \\
\mathrm{n}(\%)\end{array}$ & $\begin{array}{l}\text { Odds Ratio } \\
\text { No ajustado } \\
\text { (IC 95\%) }\end{array}$ & $\begin{array}{c}\text { Odds Ratio } \\
\text { Modelo } 1 \\
\text { Ajustado por edad } \\
\text { y sexo } \\
\text { (IC 95\%) }\end{array}$ & $\begin{array}{c}\text { Odds Ratio } \\
\text { Modelo } 2 \\
\text { Ajustado por todas } \\
\text { las variables } \\
\text { (IC 95\%) }\end{array}$ \\
\hline Edad & $53,8 \pm 8,2^{a}$ & $55,7 \pm 8,36^{\mathrm{a}}+$ & $1,03(1,02-1,04) \ddagger$ & $1,03(1,02-1,04) \ddagger$ & $1,02(1,00-1,04) \dagger$ \\
\hline Sexo masculino & $71(24,2)$ & $83(27,0)$ & $1,19(0,96-1,47)$ & $1,09(0,88-1,35)$ & $1,27(0,93-1,72)$ \\
\hline Baja educación (<8 años de educación) & $81(27,5)$ & $105(34,2)$ & $1,51(1,23-1,86) \ddagger$ & $1,35(1,08-1,69) \ddagger$ & $1,22(0,90-1,61)$ \\
\hline Bajo ingreso familiar ( $<\$ 45,000 \times$ persona/mes) & $101(34,2)$ & $114(37,0)$ & $1,04(0,86-1,27)$ & $1,05(0,85-1,28)$ & $1,34(1,02-1,76) \dagger$ \\
\hline No adherencia & $178(60,8)$ & $198(64,5)$ & $1,12(0,92-1,35)$ & $1,15(0,94-1,41)$ & $0,98(0,74-1,29)$ \\
\hline Farmacoterapia antihipertensiva múltiple & $120(40,1)$ & $162(53,2) \ddagger$ & $1,75(1,44-2,13) \ddagger$ & $1,70(1,38-2,09) \ddagger$ & $1,86(1,43-2,41) \ddagger$ \\
\hline Inadecuada relación medico-paciente & $73(23,3)$ & $91(30,1)$ & $1,41(1,13-1,79) \ddagger$ & $1,42(1,11-1,82) \ddagger$ & $1,39(1,04-1,85) \dagger$ \\
\hline Alto nivel de estres emocional-depresión & $70(24,2)$ & $97(31,6) \dagger$ & $1,36(1,10-1,68) \ddagger$ & $1,38(1,11-1,72) \neq$ & $1,56(1,14-2,12) \ddagger$ \\
\hline Escaso apoyo social & $101(34,4)$ & $115(37,3)$ & $1,14(0,94-1,39)$ & $1,10(0,90-1,34)$ & $0,95(0,72-1,26)$ \\
\hline Diabetes mellitus & $99(33,8)$ & $104(33,9)$ & $1,06(0,87-1,30)$ & $0,97(0,79-1,19)$ & $0,84(0,64-1,11)$ \\
\hline Obesidad (IMC $\geq 30$ kg/m2) & $153(52,1)$ & $198(64,4) \ddagger$ & $1,72(1,41-2,09) \ddagger$ & $1,80(1,48-2,19) \ddagger$ & $1,37(1,05-1,77) \dagger$ \\
\hline Colesterol Total $\geq 240 \mathrm{mg} / \mathrm{dL}$ & $51(17,5)$ & $80(26,1) \ddagger$ & $1,47(1,13-1,92) \ddagger$ & $1,43(1,10-1,87) \ddagger$ & $1,37(1,00-1,89) \dagger$ \\
\hline Tabaquismo (fumador actual) & $80(27,3)$ & $78(25,4)$ & $0,91(0,61-1,35)$ & $0,76(0,52-1,00)$ & $0,83(0,61-1,23)$ \\
\hline Consumo problemático de alcohol & $32(10,8)$ & $49(15,8)$ & $1,59(1,20-2,11) \ddagger$ & $1,61(1,20-2,16) \ddagger$ & $1,42(0,96-2,10)$ \\
\hline $\begin{array}{l}\text { Sedentarismo (<30 min } \times 3 \text { días/semana } \\
\text { de actividad física electiva) }\end{array}$ & $246(84,2)$ & $272(89,3)$ & $1,59(1,20-2,13) \ddagger$ & $1,52(1,14-2,04) \dagger$ & $1,17(0,81-1,69)$ \\
\hline
\end{tabular}

*media \pm desviación estándar; $\dagger p<0,05, \neq p<0,01$ 
Tabla 3. Análisis multivariado para riesgo de no adherencia a tratamiento farmacológico antihipertensivo en 600 pacientes hipertensos seguidos en el Programa de Salud Cardiovascular en la Región Metropolitana, Santiago, Chile.

\begin{tabular}{|c|c|c|c|c|c|}
\hline Variables & $\begin{array}{c}\text { Adherente } \\
\qquad \begin{array}{c}\mathrm{N}=224 \\
\mathrm{n}(\%)\end{array}\end{array}$ & $\begin{array}{c}\text { No Adherente } \\
\begin{array}{c}\mathrm{N}=376 \\
\mathrm{n}(\%)\end{array}\end{array}$ & $\begin{array}{l}\text { Odds Ratio } \\
\text { No ajustado } \\
\text { (IC 95\%) }\end{array}$ & $\begin{array}{c}\text { Odds Ratio } \\
\text { Modelo } 1 \\
\text { Ajustado por edad } \\
\text { y sexo } \\
\text { (IC 95\%) }\end{array}$ & $\begin{array}{c}\text { Odds Ratio } \\
\text { Modelo } 2 \\
\text { Ajustado por todas } \\
\text { las variables } \\
\text { (IC 95\%) }\end{array}$ \\
\hline Edad $\neq$ & $56,1 \pm 7,9$ ał & $54,4 \pm 8,3 a \ddagger$ & $0,97(0,96-098) \ddagger$ & $0,97(0,96-0,99) \ddagger$ & $0,96(0,94-0,98) \ddagger$ \\
\hline Sexo masculino & $48(21,2)$ & $112(29,7) \ddagger$ & $1,48(1,18-1,87) \ddagger$ & $1,61(1,28-2,04) \ddagger$ & $1,67(1,21-2,28) \ddagger$ \\
\hline Baja educación (<8 años de educación) & $61(26,9)$ & $127(34,0) \dagger$ & $1,29(1,04-1,60) \ddagger$ & $1,56(1,24-1,97) \ddagger$ & $1,75(1,28-2,40) \ddagger$ \\
\hline Bajo ingreso familiar ( $<\$ 45,000$ por persona/mes) & $58(26,0)$ & $157(41,9) \ddagger$ & $2,20(1,76-2,75) \ddagger$ & $2,42(1,93-3,05) \ddagger$ & $1,83(1,38-2,45) \ddagger$ \\
\hline PA no controlada (> 140/90 mmHg) & $109(48,9)$ & $198(52,7)$ & $1,12(0,92-1,35)$ & $1,15(0,93-1,41)$ & $0,93(0,71-1,23)$ \\
\hline Farmacoterapia antihipertensiva múltiple & $125(55,6)$ & $157(41,7) \ddagger$ & $0,62(0,50-0,76) \neq$ & $0,75(0,58-0,88) \ddagger$ & $0,86(0,65-1,13)$ \\
\hline Inadecuada relación medico-paciente & $45(19,9)$ & $119(31,5) \ddagger$ & $1,85(1,41-2,42) \ddagger$ & $2,17(1,64-2,86) \ddagger$ & $1,68(1,22-2,32) \ddagger$ \\
\hline Alto nivel de estrés emocional-depresión & $49(21,9)$ & $118(31,5) \ddagger$ & $1,75(1,38-2,21) \ddagger$ & $1,89(1,48-2,40) \ddagger$ & $1,92(1,36-2,71) \ddagger$ \\
\hline Escaso apoyo social & $71(31,7)$ & $145(38,5)$ & $1,28(1,04-1,57)$ & $1,29(1,04-1,59) \dagger$ & $1,10(0,83-1,49)$ \\
\hline Diabetes mellitus & $85(37,4)$ & $116(30,7)$ & $0,83(0,68-1,02)$ & $0,82(0,67-1,02)$ & $0,82(0,62-1,10)$ \\
\hline Obesidad (IMC $\geq 30$ kg/m2) & $126(56,6)$ & $225(59,7)$ & $0,99(0,81-1,21)$ & $1,00(0,82-1,23)$ & $1,03(0,78-1,35)$ \\
\hline Colesterol Total $\geq 240 \mathrm{mg} / \mathrm{dL}$ & $49(22,0)$ & $82(21,8)$ & $0,97(0,73-1,28)$ & $1,01(0,77-1,34)$ & $1,15(0,83-1,61)$ \\
\hline Tabaquismo (fumador actual) & $61(27,4)$ & $98(26,1)$ & $0,86(0,67-1,10)$ & $0,87(0,68-1,12)$ & $0,80(0,58-1,10)$ \\
\hline Consumo problemático de alcohol & $22(9,9)$ & $59(16,0) \dagger$ & $1,40(1,04-1,89) \ddagger$ & $1,14(0,84-1,55)$ & $1,36(0,87-2,13)$ \\
\hline $\begin{array}{l}\text { Sedentarismo (<30 min } \times 3 \text { días/semana de } \\
\text { actividad física electiva) }\end{array}$ & $194(86,8)$ & $324(86,6)$ & $1,01(0,75-1,36)$ & $1,08(0,80-1,45)$ & $0,82(0,56-1,23)$ \\
\hline
\end{tabular}

*media \pm desviación estándar; $\dagger p<0,05, \hbar p<0,01$

y Modelo 2. Edad, obesidad, colesterol total elevado, Rx múltiple ( $\geq$ a 2 fármacos), inadecuada relación médico paciente, elevado nivel de estrés emocional y depresión fueron asociadas a PA no controlada en ambos modelos. Baja educación, consumo problemático del alcohol y estilo de vida sedentario mostraron asociación con PA no controlada solo en Modelo 1. Bajo ingreso familiar mostró dicha asociación solo en Modelo 2. La no adherencia a Rx no mostró asociación con PA no controlada (OR 1,01, [IC 95\% 0,78-1,32], $\mathrm{p}=0,94)$.

\section{Factores asociados a la no adherencia al Rx prescrito (Tabla 3).}

Se realizó análisis de regresión logística multivariante en el subgrupo de 600 pacientes para calcular OR y evaluar el riesgo de no adherencia a Rx. La Tabla 3 muestra OR no ajustado y ajustados utilizando Modelo 1 y Modelo 2 . La no adherencia se asoció con el sexo masculino, bajo ingreso familiar, bajo nivel de educación, inadecuada re- lación médico paciente y alto nivel de estrés emocional y depresión en ambos modelos. La Rx múltiple no mostró asociación con la no adherencia después del ajuste por todas las variables (Modelo 2).

\section{Discusión}

La proporción de pacientes con control de la PA fue 56,5\% tras 1 año de seguimiento en el PSCV lo que contrasta notablemente con los resultados de la Encuesta Nacional de Salud $2010^{17}$ que indicaban que solo $16,9 \%$ de la población chilena general de hipertensos tenía su PA bajo control. Estos resultados son similares a los hallazgos del estudio ALLHAT en EE.UU ${ }^{18}$, en el que control de la PA se alcanzó en 56,2\% de los pacientes después de un año de seguimiento y en $62,7 \%$ tras 4 años. También mostró que los hispanos tenían $20 \%$ más probabilidades de lograr el control de la PA que los blancos no hispanos, revirtiendo drásticamente las estadísticas previamente descritas entre estos grupos raciales en EE.UU ${ }^{18}$. $\mathrm{Al}$ igual que en nuestro 
estudio los participantes recibieron un enfoque integral de manejo que incluye asesoramiento y educación de hábitos saludables (dieta, consumo de alcohol, tabaquismo, actividad física). Sin embargo, no se incluyó en este estudio información sobre la adherencia a tratamiento ni la influencia de los factores psicosociales.

La asociación de niveles bajos de educación e ingreso, relación médico paciente inadecuada y alto nivel de estrés emocional y depresión con control de la PA y adherencia medicamentosa observada en nuestro estudio confirma lo ya comunicado por otros en países de alto nivel de desarrollo socioeconómico ${ }^{18-21}$. Hasta donde nos ha sido posible determinarlo, no hemos encontrado información comparable proveniente de países en etapas de transición socioeconómica como Chile. La asociación de obesidad y colesterol total elevado con hipertensión no controlada encontrada en nuestro estudio coincide con lo comunicado por otros ${ }^{2,4,18}$. Asociaciones entre factores socioeconómicos y psicosociales con otras enfermedades crónicas como la diabetes y obesidad y estilos de vida no saludables también han sido documentadas ${ }^{22}$. La asociación de relación medico paciente inadecuada con hipertensión no controlada y no adherencia puede tener especial relevancia en organizaciones de salud en que se prioriza la cantidad sobre la calidad de las atenciones, o en lugares en que existe una muy limitada cantidad de proveedores ${ }^{23-25}$.

A pesar de una adherencia a Rx en sólo el 37,3\% de nuestros pacientes, 56,3\% lograron una PA controlada, y el promedio de mejoría en la PA al cabo de un año de seguimiento fue significativo (de 148,1/96,2 a 135,7/80,9 $\mathrm{mmHg}, \mathrm{p}<0,001)$. Un análisis de regresión logística multivariada de PA no controlada y no adherencia confirmó esta falta de asociación (OR 0,96 [IC 95\% 0,72 - 1,26]). En Chile no existe información previa al PSCV con respecto a la adherencia medicamentosa en los hipertensos para determinar si han existido cambios en referencia a nuestros hallazgos.

Múltiples autores que han analizado las relaciones entre la adherencia al Rx en los hipertensos con el control de la PA no han encontrado una asociación predecible $e^{9,20,26,27,28}$. Una revisión de 30 estudios en hipertensos en los que la adherencia fue evaluada a través del monitoreo electrónico de la apertura de los envases no logró encontrar una relación entre adherencia y PA registrada ${ }^{27}$. Este estudio además documentó, al igual que otros, una relación inversa entre adherencia y dosificación, con mayor adherencia en los pacientes que reciben una vez al día su medicamento ${ }^{27-29}$. En contraste, en el modelo ajustado por todas las variables (Modelo 2) no encontramos en nuestro estudio una asociación entre múltiples medicamentos antihipertensivos y no adherencia, lo que también ha sido comunicado por otros ${ }^{30,31}$. Uno de estos estudios ${ }^{31}$ contó con un número alto de hipertensos con asociación de diabetes y trastornos lipídicos, similar a nuestros hallazgos.

Diferentes métodos han sido empleados para medir la adherencia que van desde cuestionarios como el más utilizado hasta la fecha, el cuestionario Morinsky-Green-Levinne (versiones MGL-4 y MGL-8), al monitoreo de las prescripciones retiradas por los pacientes en la farmacia, conteo de las píldoras residuales en los envases durante las visitas al consultorio, hasta el monitoreo electrónico de la apertura de los envases. El cuestionario MGL ha sido ampliamente utilizado y validado hasta la fecha, pero al igual que los otros métodos está sujeto a críticas. El monitoreo electrónico es considerado como el método más confiable pero demasiado costoso para su uso habitual ${ }^{26-28}$.

La falta de una relación predecible entre la adherencia al Rx y el control de la PA es sugerente de otros factores en juego mas allá de la farmacoterapia, lo que estimamos es apoyado por nuestro estudio. Ha sido sugerido que la influencia de factores emocionales en determinar una PA no controlada o falta de adherencia puede estar mediado por vías causales del sistema nervioso central y autonómico. Un estudio clásico que midió las respuestas hemodinámicas en individuos con PA en el límite normal alto al stress emocional inducido por problemas numéricos complejos apoya esta interpretación ${ }^{32}$. Más recientemente, otros estudios han agregado evidencias adicionales al respecto ${ }^{33,34}$.

Una de las limitaciones de nuestro estudio fue el uso de un cuestionario (MGL-4) para evaluar la adherencia aunque ningún método usado hasta la fecha está libre de problemas, incluyendo el monitoreo electrónico ${ }^{20,26,29}$. Sin embargo, concordancia de $75 \%$ en la evaluación de la adherencia ha sido descrita entre el monitoreo electrónico y cuestionarios llenados por los pacientes ${ }^{35,36}$. Otra limitación es que las características psicosociales fueron medidas sólo en un subgrupo de pacientes referidos al PSCV aunque en los mismos centros de APS donde se seleccionó la muestra total y por lo tanto con similares características socioeconómicas. Finalmente la actividad física fue evaluada usando el cuestionario de la Encuesta Nacional de Salud 2010, que considera solamente la actividad física electiva sin incluir otras como la utilizada en el transporte (bicicleta, caminar) o trabajo, comunes en la población estudiada.

Para concluir, estimamos que nuestro estudio apoya la importancia de un programa integral y multidisciplinario en el manejo de los hipertensos, con acceso sin restricciones económicas a la atención médica y a un seguimiento pro- 
gramado, que aporta educación y recomendaciones para cambios en los estilos de vida desfavorables, similar a lo que proveen algunos programas existentes en países de nivel socioeconómico alto $2,3,18,26,31$. Pacientes con un grado importante de estrés y depresión se sienten emocionalmente apoyados en este tipo de programas, como ha sido sugerido por diversos autores ${ }^{21,37}$.

Los resultados de este estudio en el control de la PA pueden apoyar la consideración de estrategias similares en el manejo de los hipertensos en países con recursos limitados o que se encuentran en un proceso de transición socioeco- nómica como el de Chile.

\section{Agradecimientos}

Los autores desean agradecer la inestimable colaboración de los profesionales de la salud y personal administrativo en la realización de este estudio a los 52 centros de Atención Primaria de Salud, especialmente al CESFAM Canciller Orlando Letelier, CESFAM Cóndores de Chile, CESFAM Rosita Renard y CESFAM Edgardo Enríquez Frödden.

\section{Referencias}

1. KEARNEY PM, WHELTON M, REYNOLDS K, MUNTNER P, WHELTON PK, HE J. Global burden of hypertension: analysis of worldwide data. Lancet. 2005;365:217-223.

2. CHOBANIAN AV, BAKRIS GL, BLACK HR, CUSHMAN WC, GREEN LA, IZZO JL Jr, et al. The seventh report of the Joint National Committee on Prevention, Detection, Evaluation, and Treatment of High Blood Pressure: the JNC 7 report. JAMA. 2003;289:2560-2572

3. THE TASK FORCE FOR THE MANAGEMENT OF ARTERIAL HYPERTENSION OF THE EUROPEAN SOCIETY OF HYPERTENSION (ESH) AND OF THE EUROPEAN SOCIETY OF CARDIOLOGY (ESC). 2013 ESH/ESC Guidelines for the management of arterial hypertension. Eur Heart J 2013; 34 : 2159-2219.

4. BLOOM DE, CAFIERO ET, JANÉ-LLOPIS E, ABRAHAMS-GESSEL S, BLOOM LR, FATHIMA S, et al. The global economic burden of non-communicable diseases. Geneva: World Economic Forum (2011).

5. MARMOT M. Social Determinants of Health Inequalities. Lancet 2005;365:1099-1104.

6. SANDOVAL D, BRAVO M, KOCH E, GATICA S, AHLERS I, HENRIQUEZ O, et al. Overcoming barriers in the management of hypertension. The experience of the Cardiovascular Health Program in Chilean Primary Health Care Centers. Int J Hypertens. Volumen 2012.

7. INSTITUTO NACIONAL DE ESTADISTICA. Estadísticas Vitales. Anuario 2012. Santiago de Chile, Septiembre 2012.
8. INSTITUTO NACIONAL DE ESTADISTICA. Primer estudio de la discapacidad en Chile. Santiago de Chile, Abril 2005.

9. GARRIDO J, CHACON J, SANDOVAL D, MUÑOZ R, LOPEZ N, OYARZUN E, et al. Control del hipertenso un desafío no resuelto. Avances logrados en Chile mediante el Programa de Salud Cardiovascular. Rev Chil Cardiol 2013; 32:85-96.

10. MORISKY DE, GREEN LW, LEVINE DM. Concurrent and predictive validity of a self-reported measure of medication adherence. Med Care 1986; 24: 67-74.

11. MORISKY DE, ANG A, KROUSEL-WOOD M, WARD HJ. Predictive validity of a medication adherence measure in an outpatient setting. J Clin Hypertens 2008;10:348-354.

12. SHERBOURNE CD, STEWART AL. The MOS social support survey. Soc Sci Med 1991;32: 705-714.

13. GOLBERG DP, HILLIER VF. A scaled version of the General Health Questionnaire. Psychol Med 1979; 9: 139-145.

14. BOZZO G, MARTINEZ B, SEPULVEDA P, CARVACHO C, RIVERA G, MOORE P, et al. Development and validation of a survey for patients satisfaction in primary care clinics. Rev Med Chile 1995;123:1160-1164.

15. FLORENZANO E, HORWITZ N, PENNA M, VALDES M. Validation of an abbreviated scale for detection of abnormal drinking behavior (E.B.B.A) (Escala Breve para la detección del Beber Anormal). Mental Health and Primary Health Care subjects (Temas de Salud Mental y Atención Primaria de Salud.) Santiago: Facultad de Medicina, Universidad de Chile. 1991: 185-93. 
16. MINISTERIO DE SALUD. Primera Encuesta Nacional de Salud. Informe Final, Santiago, Chile, 2003.

17. MINISTERIO DE SALUD. Encuesta Nacional de Salud. Tomo I. Santiago, Chile, 2009-2010.

18. MARGOLIS KL, PILLER LB, FORD CF, HENRIQUEZ MA, CUSHMAN WC, EINHORN PT, et al. Blood pressure control in hispanics in the antihypertensive and lipid-lowering treatment to prevent heart attack trial. Hypertension 2007;50:854-861.

19. VALDERRAMA AL, GILLESPIE C, MERCADO C. Racial/ ethnic disparities in the awarenes, treatment and control of hypertension, United States, 2003-2010 centers for disease control and prevention. MMWR 2013; 62:352-54.

20. CHOBANIAN AV. The hypertension paradox-more uncontrolled disease despite improved therapy. N Engl J Med 2009; 361:878-88.

21. EZE-NLIAM CM, THOMBS BD, LIMA BB, SMITH CG, ZIEGELSTEIN RC. The association of depression with adherence to antihypertensive medications: a systematic review. J Hypertens 2010; 28:1785-95.

22. DUNBAR-JACOB J, ERLEN JA, SCHLENK EA, RYAN CM, SEREIKA SM, DOSWELL WM. Adherence in chronic disease. Annu Rev Nursing Res 2000; 18: 48-90.

23. BERLOWITZ DR, ASH AS, HICKEY EC, FRIEDMAN RH,GLICKMAN M, KADER B, et al. Inadequate management of blood pressure in a hypertensive population. N Engl J Med 1998; 339:1957-1963.

24. PHILLIPS LS, BRANCH WT, COOK CB, DOYLE JP, EL-KEBBII M, GALLINA DL et al. Clinical inertia. Ann Intern Med 2001; 135: 825-834.

25. DIEZ ROUX AV. Persistent social patterning of cardiovascular risk: rethinking the familiar. Circulation 2005; 111: 3020-3021.

26. PICKERING TG. Why are we doing so badly with the control of hypertension? Poor compliance is only part of the story. J Clin Hypertens 2001; 3: 179-82.

27. WETZELS GEC, NELEMANS P, SCHOUTEN JS, PRINS $\mathrm{MH}$. Facts and fiction of poor compliance as a cause of inadequate blood pressure control: a systematic review. J Hypertens 2004;22:1849-1855.
28. SCHROEDER K, FAHEY T, HAY AD, MONTGOMERY A, PETERS T. Relationship between medication adherence and blood pressure in primary care: a prospective study. J Hum Hypertens 2006; 20: 625-7.

29. BRAMLEY TJ, GERBINO PP, NIGHTENGALE BS, FRECH-TAMAS F. Relationship of blood pressure control to adherence with antihypertensive monotherapy in 13 managed care organizations. J Manag Care Pharm 2006; 12: 239-45.

30. CLAXTON AJ, CRAMER J, PIERCE C. A systematic review of the associations between dose regimens and medication compliance. Clin Ther 2001; 23: 1296-310.

31. MAZZAGLIA G, AMBROSIONI E, ALACQUA M, FILIPPI A, SESSA E, IMMORDINO V, et al. Adherence to antihypertensive medications and cardiovascular morbidity among newly diagnosed hypertensive patients. Circulation 2009; 120: 1598-1605.

32. SHULTE W, HEUS H. Hemodynamics during emotional stress in borderline and mild hypertension. Eur Heart J 1983; 4: 803809.

33. TSIGOS C, CHROUSOS GP. Hypothalamic-pituitary-adrenal axis, neuroendocrine factors and stress. J Psychosom Res 2002; 53: $865-871$.

34. ROSE KM, NORTH K, ARNETT DK, ELLISON RC, HUNT SC, LEWIS CE, et al. Blood pressure and pulse responses to three stressors: Associations with sociodemographic characteristics and cardiovascular risk factors. J Hum Hypertens 2004; 18: 333-341.

35. CHOO PW, RAND C, INUI TS, LEE ML, CAIN E, CORDEIRO-BREAULT M, et al. Validation of patient reports, automated pharmacy records, and pill counts with electronic monitoring of adherence to antihypertensive therapy. Med Care 1999; 37: 846-867.

36. GARBER MC, NAU D, ERICKSON SR, AIKENS JE, LAWRENCE JB. The concordance of self-report with other measures of medication adherence: A summary of the literature. Med Care 2004; 42: 649-52.

37. WU EL, CHIEN IC, LIN CH, CHOU YJ, CHOU P. Increased risk of hypertension in patients with major depressive disorder: A population-based study. J Psychosom Res 2012; 73:169-74. 